\title{
http://bjas.journals.ekb.eg \\ Updates in perioperative Assessment of Acute Renal Impaired Patient
}

\author{
M.Y.Serry, M.H.Abd-Elrahman and A.H.Badran
}

Anesthesia and surgical ICU Dept., Faculty of Medicine, Benha Univ., Benha, Egypt

E-Mail: Mohamed@gmail.com

\begin{abstract}
Perioperative acute kidney injury (AKI) is more common than previously recognized, especially in high-risk patients undergoing higher risk procedures. The developing number of patients who create perioperative AKI is connected, to a limited extent, to the maturing populace and increment in the quantity of people with interminable comorbidities, especially those with premorbid incessant kidney malady. In spite of the acknowledgment of normalization in the meaning of AKI, clinicians routinely underdiagnose it and neglect to value that it is related with impressive grimness and mortality. Suitable administration of intravenous liquid substitution is a key part of the treatment of intense kidney injury (AKI). In patients with intense glomerulonephritis and other inborn renal maladies, there is minimal clinical debate that sodium and water limitation is valuable in the setting of impeded renal excretory capacity. Then again, in patients with AKI confusing foundational ailment, supplemental intravenous liquids are viewed as a basic component of treatment. It is significant, in any case, to think about the physiological basis of liquid treatment to forestall both under treatment and unnecessary volume development. The mix of the distinctive scoring models firmly bolsters and profoundly improves the prognostic execution of either model alone. In this way, we do suggest the joined utilization of APACHE II score, SOFA score along with RIFLE score for forecast of mortality of fundamentally sick patients in emergency unit.
\end{abstract}

Keywords: Perioperative, Acute renal impaired, AKI.

\section{Introduction}

The frequency of perioperative intense kidney injury (AKI) is more typical than recently perceived, particularly in high-chance patients experiencing higher hazard methods. The developing number of patients who create perioperative AKI is connected, to a limited extent, to the maturing populace and increment in the quantity of people with ceaseless comorbidities, especially those with premorbid constant kidney illness. Regardless of the acknowledgment of normalization in the meaning of AKI, clinicians routinely underdiagnose it and neglect to value that it is related with significant grimness and mortality [1] .

Tragically, hardly any, preemptive treatments have demonstrated viable in forestalling AKI. Auspicious indicative techniques utilizing developing biomarkers raises the possibility of location of kidney harm before the beginning of irreversible loss of capacity, yet stay under scrutiny. Clear proof supporting any helpful mediation aside from renal substitution treatment stays subtle. Renal swap treatment is demonstrated for select patients with dynamic AKI; be that as it may, the perfect planning, strategy, and use of it stay under discussion. It is principal to distinguish patients in danger for AKI. The Kidney Disease: Improving Global Outcomes rules recommend preventive methodologies that incorporate shirking of nephrotoxic operators and hyperglycemia, enhancement of hemodynamics, rebuilding of the coursing volume, and organization of utilitarian hemodynamic checking. Away from on the side of this methodology, be that as it may, is inadequate [1].

As of late, the perioperative organization of dexmedetomidine and the arrangement of remote ischemic preconditioning have been concentrated as far as possible the improvement of perioperative AKI.
This audit examines acknowledged standard meanings of AKI, features related hazard factors for its turn of events, and gives an outline of its the study of disease transmission and pathology. It accentuates potential preventive systems, the conceivable job of developing biomarkers in characterizing its essence all the more speedily before irreversible injury, and current suggested rules and helpful methodologies. A definitive objective of this article is to bring to the consideration of clinicians the reality of this possibly preventable or modifiable perioperative confusion [1].

\section{Methods}

This is a review article, The search was performed in MEDLINE, Embase, Pubmed and CINAHL Plus in the same date range with the following mediacl terms: "perioperative; acute renal impaired; AKI", including articles from 2000 to 2019, Excluded articles from review are those of langauge other than English. Key words: perioperative; acute renal impaired; AKI.

\section{Results}

The occurrence of perioperative intense kidney injury (AKI) is more typical than recently perceived, particularly in high-hazard patients experiencing higher hazard techniques. The developing number of patients who create perioperative AKI is connected, to a limited extent, to the maturing populace and increment in the quantity of people with ceaseless comorbidities, especially those with premorbid constant kidney infection. In spite of the acknowledgment of normalization in the meaning of AKI, clinicians routinely underdiagnose it and neglect to value that it is related with impressive dreariness and mortality. Sadly, hardly any, preemptive treatments have demonstrated powerful in forestalling AKI. 
An expected $2 \%-18 \%$ of hospitalized patients and somewhere in the range of $22 \%$ and $57 \%$ of emergency unit create AKI during their clinic affirmation. AKI doesn't generally advance to renal disappointment requiring renal substitution treatment (RRT). Full recuperation to benchmark capacity may not happen. Indeed, even little intense increments in creatinine focus, generally saw as unimportant, can bring about short-and long haul complexities including contaminations and dying, incessant kidney sickness (CKD), end-stage renal illness, cardiovascular ailments, and passing.

A grouping framework ought to in this manner incorporate and recognize gentle or extreme, and early or late cases. This would permit such an order to distinguish patients in whom renal capacity is somewhat influenced (high affectability for the identification of kidney breakdown) and patients in whom renal capacity is extraordinarily influenced (high particularity for genuine renal brokenness).

The impressive dreariness and mortality related with perioperative intense kidney injury (AKI) is very much reported, however AKI stays an underdiagnosed and misjudged sickness. The improvement of agreement rules (Risk, Injury, Failure, Loss, End Stage Renal Disease [RIFLE] models [2004]; Acute Kidney Injury Network [AKIN] measures [2007]; Kidney Disease Improving Global Outcomes [KDIGO] rules [2012]) has caused more to notice this genuine clinical condition. AKI has been analyzed progressively in the course of recent decades.

Despite the fact that the etiology of AKI in basically sick patients is likely frequently multifactorial, sepsis is reliably been seen as a significant contributing element. A few examinations have indicated that roughly $40 \%$ to half of patients with AKI on introduction to an ICU have corresponding sepsis and that around $11 \%$ to $64 \%$ of patients with a finding of serious sepsis or septic stun have attending AKI.

Difference prompted nephropathy (CIN) has become a significant reason for iatrogenic intense renal disability. Truth be told, CIN is the third driving reason for new intense kidney injury in hospitalized patients and the frequency in ICU about $11.5 \%$.

Auspicious analytic techniques utilizing advancing biomarkers raises the possibility of recognition of kidney harm before the beginning of irreversible loss of capacity, however stay under scrutiny. Clear proof supporting any restorative mediation with the exception of renal substitution treatment stays tricky. Renal trade treatment is demonstrated for select patients with dynamic AKI; be that as it may, the perfect planning, technique, and use of it stay under discussion. It is major to recognize patients in danger for AKI.

Fitting administration of intravenous liquid substitution is a key part of the treatment of intense kidney injury (AKI). In patients with intense Table (1) The RIFLE criteria [2]. glomerulonephritis and other inherent renal ailments, there is minimal clinical debate that sodium and water limitation is useful in the setting of disabled renal excretory capacity. On the other hand, in patients with AKI convoluting foundational disease, supplemental intravenous liquids are viewed as a basic component of treatment. It is significant, nonetheless, to think about the physiological reason of liquid treatment to forestall both under treatment and extreme volume extension.

With the exception of in instances of outrageous acidemia, sodium bicarbonate ought to be administered as an implantation (over a time of a few minutes to a couple of hours) as opposed to a bolus or give just a large portion of the portion as an IV bolus, the rest by persistent IV mixture (in 5\% dextrose superior to saline to stay away from hypernatremia) more than 12-24 h. Follow-up observing of the patient's corrosive base status will decide extra salt prerequisites. About30 minutes must slip by after the mixture of bicarbonate is finished before its clinical impact can be judged.

In patients with cardiogenic stun and vasodilatory stun support of a sufficient mean blood vessel pressure and heart yield is principal to guarantee satisfactory fundamental organ perfusion and capacity. In numerous ICU patients, plasma volume extension is adequate to accomplish these objectives. In numerous others it isn't. In these patients, vasoactive medications (a large number of which have both inotropic and vasopressor properties) are utilized to expand either heart yield or perfusion pressure, or both. Be that as it may, numerous parts of their utilization stay questionable. One specific zone of discussion identifies with their renal impacts.

AKI doesn't generally advance to renal disappointment requiring renal substitution treatment (RRT). Full recuperation to standard capacity may not happen. Indeed, even little intense increments in creatinine focus, generally saw as paltry, can bring about short and long haul entanglements including contaminations and dying, constant kidney infection (CKD), end-stage renal ailment, cardiovascular illnesses, and demise. It is imperative that advancement or movement of constant issue after a scene of AKI has major financial and general wellbeing impacts.

The blend of the distinctive scoring models emphatically underpins and profoundly improves the prognostic execution of either model alone. In this way, we do suggest the consolidated utilization of APACHE II score, SOFA score along with RIFLE score for expectation of mortality of fundamentally sick patients in emergency unit.

RIFLE characterization is as reliable as other scoring framework like SOFA, APACHE II, and MODS in regards to mortality expectation in patients with sepsis and SIRS gave intense renal shutdown 


\begin{tabular}{|c|c|c|}
\hline & GFR criteria & Urine output criteria \\
\hline Risk & Cr increased $1.5 x$ or GFR decrease $>25 \%$ & $<0.5 \mathrm{ml} / \mathrm{kg} / \mathrm{h}$ for $6 \mathrm{hrs}$ \\
\hline Injury & $\mathrm{Cr}$ increased 2.0x or GFR decrease $>50 \%$ & $<0.5 \mathrm{ml} / \mathrm{kg} / \mathrm{h}$ for $12 \mathrm{hrs}$ \\
\hline Failure & $\begin{array}{c}\text { Cr increased 3.0x or GFR decrease }>75 \% \\
\text { or } \mathrm{Cr} \geq 4 \mathrm{mg} / \mathrm{dL} \text { when there was an acute } \\
\text { rise of }>0.5 \mathrm{mg} / \mathrm{dl}\end{array}$ & $\begin{array}{l}<0.3 \mathrm{ml} / \mathrm{kg} / \mathrm{h} \text { for } 24 \mathrm{hrs} \text { or anuria } \\
\text { for } 12 \mathrm{hrs}\end{array}$ \\
\hline Loss & \multicolumn{2}{|c|}{ Persistent ARF; complete loss of kidney function for $>4$ weeks } \\
\hline $\begin{array}{l}\text { End-stage } \\
\text { renal disease }\end{array}$ & \multicolumn{2}{|c|}{ ESRD for 3 months } \\
\hline
\end{tabular}

\section{Discussion}

The idea of intense renal disappointment (ARF) has experienced huge reconsideration as of late. Mounting proof recommends that intense, moderately mellow brokenness of the kidney, show by changes in pee yield and blood sciences, forecasts genuine clinical outcomes. In spite of the fact that the term intense renal disappointment is moderately new, its first depiction as ischuriarenalis was by William Heberden in 1802. During the First World War the condition was named "War Nephritis" and was accounted for in a few distributions. The condition was then to a great extent overlooked until the Second World War, when Bywaters and Beall distributed their old style paper on smash disorder. It is Homer W. Smith who is credited for the presentation of the term intense renal disappointment (1951) [3].

Intense kidney injury is commonly characterized as a sudden and supported decline in kidney work'. Up to this point there has not been an agreement on how best to survey kidney work; in particular, what markers best reflect kidney work, and what estimations of those markers separate typical from irregular kidney work [2] .

The reasons why little changes in kidney work lead to increments in emergency clinic mortality are indistinct. Potential clarifications incorporate the undesirable impacts of intense kidney brokenness, for example, volume over-burden, maintenance of uremic mixes, acidosis, electrolyte issue, expanded hazard for disease, and paleness [4].

Alongside contrasts in tolerant qualities, this absence of consistency in the analysis has most likely added to the wide variety in the announced rate and result of ARF (occurrence goes somewhere in the range of 1 and $31 \%$ and mortality is somewhere in the range of 28 and $82 \%$ [5].

Terms, for example, intense renal disappointment, intense kidney ailment, intense kidney disorder, or AKI have been utilized in the past to depict kidney brokenness emerging from intense conditions. AKI analysis and research was tormented by many extraordinary and conflicting definitions. Eventually, in 2004, the Acute Dialysis Quality Initiative discharged the RIFLE standards, which supplanted the expression "intense renal disappointment" and presented the expression "AKI" to show the reversibility of the intense condition. Along these lines, the RIFLE rules quickly increased wide acknowledgment to characterize AKI. They depended on changes in serum creatinine $(\mathrm{sCr})$ from standard or pee yield. The analytic framework separates 3 seriousness grades (hazard, injury, disappointment) and 2 result classes (misfortune, end-stage renal illness). At the hour of its presentation, the idea of AKI was alluring in light of the fact that it included patients without real auxiliary kidney harm yet with practical weakness. This gathering of patients may profit essentially from early helpful intercessions. In spite of qualities of the RIFLE characterization, the conspicuous error between increments in $\mathrm{SCr}$ fixation and diminishes in evaluated glomerular filtration rate (GFR) stayed risky. In 2007, the AKIN recommended an adjustment of the RIFLE models, trying to improve their affectability. The bar for diagnosing AKI was fundamentally brought down. In 2012, the Acute Kidney Injury Working Group of KDIGO discharged the most recent characterization framework with the point of binding together the RIFLE and AKIN rules [6].

The first stratum of the RIFLE criteria (risk) might be the most important one, because at this stage, a positive test should increase the physician's awareness of the presence of risk for renal injury, at a moment when the situation still is reversible by preventive or therapeutic intervention (Hoste EA et al., 2006).

\subsection{Risk Factors for the Development of AKI}

A number of risk factors predispose for AKI. However, patient-related factors are more strongly associated with mortality than the type of surgery.

\subsection{Diagnosis of AKI}

Early detection of the occurrence of acute kidney injury (AKI) and delineation of the cause (s) of this disorder are important to start early therapy and perhaps reduce morbidity and mortality. So diagnosis of AKI needs thorough work up, which includes:

I) History and physical examination

II) Investigations:

A. Laboratory: urine analysis, diagnostic indices, biomarkers

B. Imaging and radiological investigations.

C. Renal biopsy

D. Other confirmatory tests for differential diagnosis of AKI. 
AKI diagnostic criteria. KDlGO crite ia for the drafosis of AKL. AKI indicates acute Disease: Improving CRT, renal replacement therapy: $\mathrm{s} C$, serum creatinine; UO, urine output.
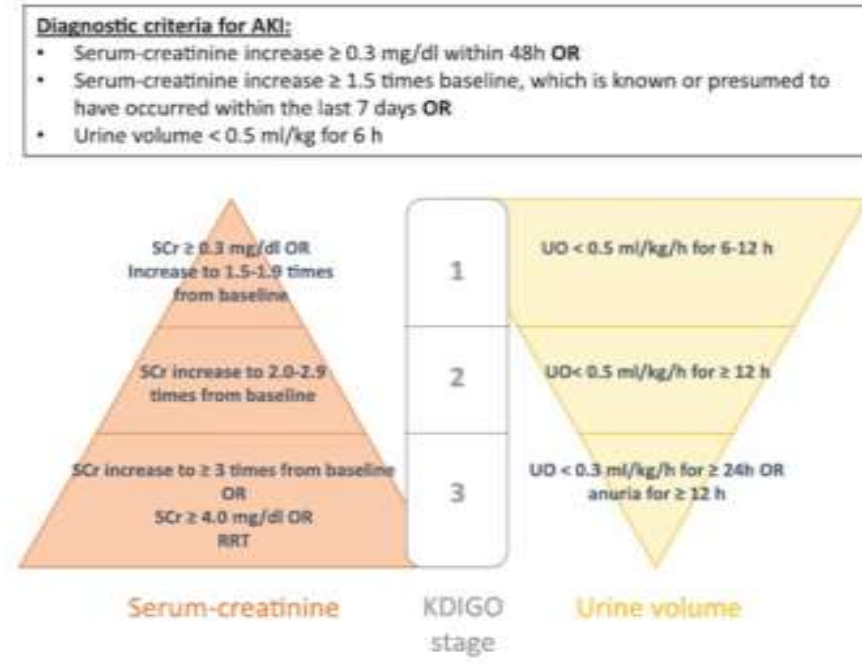

Fig (1) Diagnostic criteria for AKI [7]

Table (2) Risk factors for perioperative AKI [8].

\begin{tabular}{lc}
\hline Preoperative risk factors & Intraoperative risk factors \\
\hline Age & Duration of surgery \\
Female sex & Intraperitoneal surgery \\
Body mass index & Repair of abdominal aortic aneurysm \\
Hypertension & Intraoperative hypotension \\
Chronic kidney disease & Transplantation of solid non-renal \\
Insulin-requiring diabetes mellitus & Transfusion of packed red blood cells \\
Chronic obstructive pulmonary disease & Intraabdominal hypertension \\
Peripheral vascular disease & Length of cardiopulmonary bypass ( cardiac \\
& surgery ) \\
Cerebrovascular disease & Cross- clamp time ( cardiac surgery ) \\
Congestive heart failure & Hemodilution ( cardiac surgery ) \\
Sepsis & Use of intraaaortic ballon pump \\
Ascites & Type of cardiac surgical procedure nephrotoxic \\
& agents) eg, antibiotics ,contrast agents ) \\
\hline
\end{tabular}

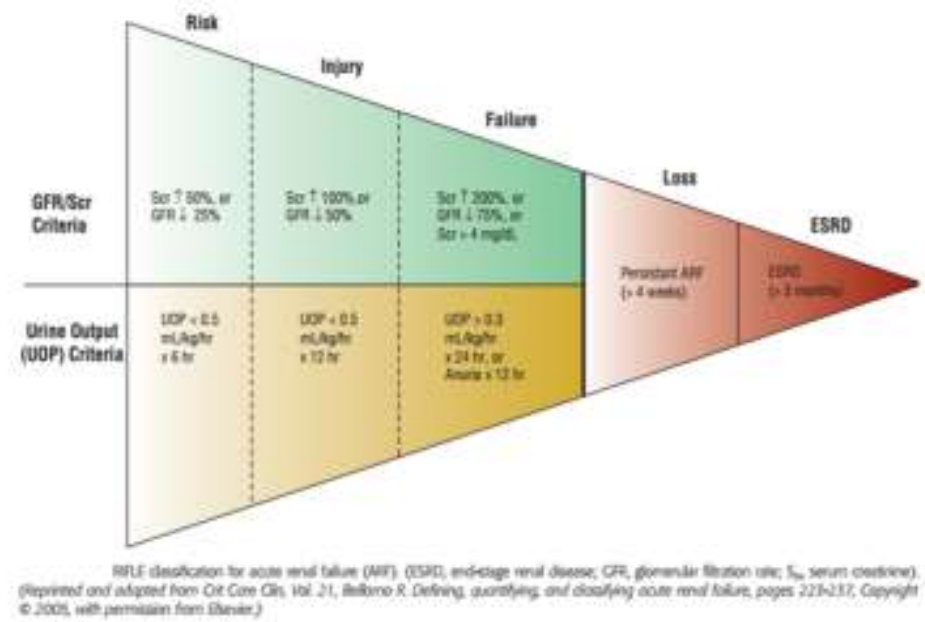

Fig (2) RIFLE CRITERIA [9].

Roughly $30 \%-40 \%$ of all instances of AKI cases happen after medical procedure. The frequency of
AKI in careful patients ranges from $18 \%$ to $47 \% .26$ 28 Some careful patient populaces merit extraordinary 
thought since they are presented to particular hazard factors innate to the sort of medical procedure. AKI is generally normal among cardiovascular medical procedure patients, in whom cardiopulmonary detour (CPB) is utilized. These patients face a one of a kind mix of renal stressors. Paces of AKI for elective patients are in the scope of $15 \%$, with $2 \%$ requiring RRT. Yet in addition in noncardiac medical procedure, AKI happened in roughly $1 \%$ of those patients with preoperative ordinary kidney work. Certain noncardiac medical procedure methodology represent a specific high hazard:

- gastric sidestep medical procedure for bleak stoutness is related with a $8.5 \%$ occurrence of AKI;

- one-third of the patients getting liver transplant experience postoperative AKI, with $17 \%$ requiring RRT;

- patients with previous CKD demonstrated a fundamentally expanded AKI chance than the normal $1 \%$. Patients who create AKI throughout medical procedure exhibit a 8-overlay expanded hazard for the movement to CKD [10].

In basically sick patients over $90 \%$ of ARF scenes are of ischemic or poisonous etiology or a mix of both. It is generally connected with brokenness of other organ frameworks, is frequently joined by sepsis and is regularly multi-factorial. Septic ARF is the most incessant and deadly reason for kidney disappointment in the emergency unit). In any case, the exact finding of the etiology of ARF isn't generally clear or simple to set up [11].

Notwithstanding the hidden reason, AKI is related with not just essentially expanded in-clinic grimness, mortality, and expenses yet additionally with a higher mortality hazard in patients who made due for in any event $90 \mathrm{~d}$ after emergency clinic release [11] .

The level of patients with ARF who require renal substitution treatment (RRT) ranges from 20 to $60 \%$. Among the subgroup of patients who endure beginning dialysis, under $25 \%$ require long haul dialysis, showing the potential reversibility of the condition [11].

Huge advancement has been made in the field of RRT for fundamentally sick patients with AKI in the course of the most recent three decades. The treatment choices have extended from essential intense peritoneal dialysis and discontinuous hemodialysis (IHD), to now incorporate an assortment of nonstop modalities (CRRT), going from hemofiltration, dialysis as well as hemodiafiltration, and an assortment of cross breed treatments, differently portrayed as expanded every day dialysis as well as hemodiafiltration, with the chance of extra aide treatments including plasma division and adsorption methods [12] .

Late informationon the definition, the study of disease transmission, pathophysiology, and clinical reasons for AKI in the ICUs is a principal essential for counteraction of this issue. A multidisciplinary approach, which nephrologist and intensivist work one next to the other to accomplish ideal consideration for a given patient, is basic for effective administration. The misperception that nephrologists have little to offer restoratively with the exception of RRT additionally may postpone proper counsel, permitting uremic intricacies and further kidney injury to happen [12].

One investigation indicated that postponed nephrology discussion was related with expanded mortality and expanded length of medical clinic and emergency unit stays [13].

AKI doesn't generally advance to renal disappointment requiring renal substitution treatment (RRT). Full recuperation to pattern capacity may not happen. Indeed, even little intense increments in creatinine focus, truly saw as minor, can bring about short and long haul complexities including contaminations and dying, interminable kidney infection (CKD), end-stage renal ailment, cardiovascular maladies, and passing. It is essential that improvement or movement of constant issue after a scene of AKI has major financial and general wellbeing impacts [12].

\section{Conclusion}

Perioperative AKI continues to be a feared consequence of surgery. It is associated with both short- and long-term deleterious effects. The complexity of perioperative AKI pathophysiology involves the combined roles of ischaemia and inflammation as causes of AKI. Novel biomarkers are proposed as a faster and more accurate way for prompt identification of AKI and could prove beneficial in early intervention to prevent further deterioration in renal function. Specific comorbidities, surgeries, and interventions increase the risk of AKI, such as cardiac or transplant surgery and use of contrast dye. The intraoperative period is unique in that both anaesthesia and surgery combine to affect renal function. During surgery, even short periods of hypotension put the kidney at risk. Urine output does not predict postoperative AKI. Careful selection and use of i.v. fluids and vasopressors and appropriate blood management are important to prevent perioperative AKI.

\section{References}

[1] D. P. Basile, "Progression after AKI: understanding maladaptive repair processes to predict and identify therapeutic treatments," J. Am. Soc. Nephrol, Vol.27(3), PP. 687-697, 2016.

[2] R. Bellomo, C. Ronco, J. A. Kellum, “Acute renal failure-definition, outcome measures, animal models, fluid therapy and information technology needs: the Second International Consensus Conference of the Acute Dialysis Quality Initiative (ADQI) Group," Crit. care, Vol.8(4), p. R204, 2004.

[3] D. A. Molony , J. C. Craig, Evidence-based 
nephrology, John Wiley \& Sons, Vol.71 (1), PP. 32-39, 2011.

[4] E. A. Hoste , J. A. Kellum, "Acute renal failure in the critically ill: impact on morbidity and mortality," in Sepsis, Kidney and Multiple Organ Dysfunction, Karger Publishers, Vol.144 (1), PP. 1-11, 2004.

[5] H. Schiffl, S. M. Lang , R. Fischer, "Daily hemodialysis and the outcome of acute renal failure," N. Engl. J. Med, Vol.346 (5), PP. 305$310,2002$.

[6] E. A. J. Hoste, "Epidemiology of acute kidney injury in critically ill patients: the multinational AKI-EPI study," Intensive Care Med, Vol.41( 8), PP. 1411-1423, 2015.

[7] I. Baldwin, R. Bellomo, T. Naka, "A pilot randomized controlled comparison of extended daily dialysis with filtration and continuous venovenous hemofiltration: fluid removal and hemodynamics," Int. J. Artif. Organs, Vol.30(12), PP. 1083-1089, 2007.

[8] M. E. Grams, "Acute kidney injury after major surgery: a retrospective analysis of veterans health administration data," Am. J. Kidney Dis, Vol.67(6), PP. 872-880, 2016.
[9] E. A. J. Hoste, "RIFLE criteria for acute kidney injury are associated with hospital mortality in critically ill patients: a cohort analysis," Crit. care, Vol.10(3), p. R73, 2006.

[10] W. K. Han, V. Bailly, R. Abichandani, "Kidney Injury Molecule-1 (KIM-1): a novel biomarker for human renal proximal tubule injury," Kidney Int, Vol.62(1), PP. 237-244, 2002.

[11] M. K. Nadim, "Impact of the etiology of acute kidney injury on outcomes following liver transplantation: acute tubular necrosis versus hepatorenal syndrome," Liver Transplant, Vol.18(5), PP. 539-548, 2012.

[12]L. S. Chawla, R. L. Amdur, A. D. Shaw, "Association between AKI and long-term renal and cardiovascular outcomes in United States veterans," Clin. J. Am. Soc. Nephrol, Vol.9 (3), PP. 448-456, 2014.

[13]R. L. Mehta, W. C. Clark, M. Schetz, "Techniques for assessing and achieving fluid balance in acute renal failure," Curr. Opin. Crit. Care, Vol.8 (6), PP. 535-543, 2002. 